The University of San Francisco

USF Scholarship: a digital repository @ Gleeson Library | Geschke Center

Nursing and Health Professions Faculty Research and Publications

School of Nursing and Health Professions

2013

\title{
Self-Management of Unpleasant Auditory Hallucinations: A Tested Practice Model
}

Robin Buccheri

University of San Francisco, buccherir@usfca.edu

L Trygstad

M Buffum

P Birmingham

G Dowling

Follow this and additional works at: http://repository.usfca.edu/nursing_fac

Part of the Nursing Commons, and the Psychiatric and Mental Health Commons

\section{Recommended Citation}

Buccheri R., Trygstad L., Buffum M., Birmingham P., Dowling G.(2013). Self-Management of Unpleasant Auditory Hallucinations: A Tested Practice Model. J Psychosoc Nurs Ment Health Serv. 51(11) 26-34. doi: 10.3928/02793695-20130731-02

This Article is brought to you for free and open access by the School of Nursing and Health Professions at USF Scholarship: a digital repository @ Gleeson Library | Geschke Center. It has been accepted for inclusion in Nursing and Health Professions Faculty Research and Publications by an authorized administrator of USF Scholarship: a digital repository @ Gleeson Library | Geschke Center. For more information, please contact repository@usfca.edu. 


\section{Self-Management of Unpleasant Auditory Hallucinations: \\ A Tested Practice Model}

Auditory hallucinations (AH) are often defined as hearing voices or sounds in the absence of actual or reality-based stimuli. AH can be a symptom of a mental disorder or neurological disorder but can also occur in healthy individuals (Romme \& Escher, 1989; Romme, Escher, Dillon, Corstens, \& Morris, 2009; Shinn et al., 2012). AH are viewed by voice hearers as either pleasant or unpleasant. Many people hear only pleasant AH, others hear only unpleasant AH and many hear both.

Pleasant $\mathrm{AH}$ are often harmless and can keep voice hearers company by talking to them in a nonthreatening manner or even sometimes laughing at their jokes. Unpleasant AH can be extremely distressing by saying critical or derogatory things about the voice hearer, giving them bad advice or commanding them to harm themselves or someone else. Voice hearers often want to keep their pleasant voices but would like their unpleasant voices to go away. Not surprisingly, they are more likely to accept help in learning how to self-manage their unpleasant voices.

To date there have been no practice models to help psychiatric-mental health nurses care for patients who hear unpleasant $\mathrm{AH}$. The purpose of this paper is to describe a tested practice model for teaching self-management of unpleasant $\mathrm{AH}$.

\section{Usefulness of the Model}

This paper presents the first practice model to help psychiatric-mental health nurses implement evidence-based practice into the nursing care of people with unpleasant AH. This model provides evidence-based psychiatric nursing assessments, individual and group interventions and standardized tools to evaluate those interventions. Use of 
this model helps to establish a common language of AH among staff, patients, patients' families and community caregivers. This model also assists nurses in understanding the complexity of unpleasant $\mathrm{AH}$ and the similarities and differences in how individuals experience and manage this often-distressing symptom.

Psychiatric-mental health nurses across a variety of clinical inpatient and outpatient mental health settings can use this practice model for teaching voice hearers selfmanagement strategies. This model also provides a framework for teaching nursing students and new psychiatric-mental health nurses how to interact therapeutically with those who hear unpleasant $\mathrm{AH}$.

\section{Background}

\section{Evolution of the Model}

Our practice model evolved from five main sources: 1) clinical knowledge from working in many different settings (e.g., inpatient, outpatient, support group) with people who experience unpleasant $\mathrm{AH} ; 2$ ) theoretical knowledge from symptom management theory (Dodd, et al., 2001; Humphreys, et al., 2008; UCSF, 1995), Brier and Strauss' (1983) application of Kanfer's framework on self-control of psychotic symptoms; psychosocial recovery model (Deegan, 1973), principles of adult learning theory (Knowles, 1973; 1998), and the peer counselor movement (Davidson et al., 1999); 3) patient outcomes after participation in our 10-session Behavioral Management of Auditory Hallucinations Course (Buccheri et al., 2004; Buccheri, Trygstad \& Dowling, 2007; Buccheri, Trygstad, Kanas, \& Dowling, 1997; Buccheri, Trygstad, Kanas, Waldron, \& Dowling, 1996; Trygstad et al., 2002); 4) course facilitator feedback after teaching the 10-session course and patient feedback after attending the course (Buffum et 
al., 2009); and 5) advocacy organizations such as National Alliance of the Mentally Ill (NAMI) and Intervoice.

\section{Description of Self-Management of}

\section{Unpleasant Auditory Hallucinations Practice Model}

The self-management of unpleasant auditory hallucinations practice model guides the nursing care of people who suffer from unpleasant $\mathrm{AH}$. There are three parts to the model: Assessment of Voice Hearer's Experience, Nursing Interventions, and Voice Hearer's Outcomes. Figure 1 displays the entire model.

\section{Assessment of Voice Hearer's Experience}

The first part of the model is comprised of three author-developed and tested instruments that, used together, provide a comprehensive assessment of each voice hearer's experience with AH. The information from this assessment can be used to create an individualized care plan for each voice hearer.

These three instruments are the: Auditory Hallucinations Interview Guide (AHIG), Unpleasant Voices Scale (UVS/0-10) and Characteristics of Auditory Hallucinations Questionnaire (CAHQ). The AHIG is used for assessment and the other two instruments (CAHQ and UVS) are used to assess symptoms and to measure outcomes (symptom changes over time). Each instrument is described below.

The AHIG is a 32-item instrument that mental health clinicians can use to interview voice hearers. It takes approximately 30 minutes to complete and asks detailed questions about the onset of their voices and their experience hearing voices (e.g., How old were you when you first started hearing voices?; What is the most distressing part of hearing voices?; Do you ever hear sounds other than voices?). 
The 7-item CAHQ measures 7 characteristics of auditory hallucinations: frequency, loudness, self-control, clarity, tone, distractibility and distress on a scale of 0 (no voices heard) to 5 (the most negative rating; for example, a 5 rating for "frequency" is interpreted as "constantly") (Buccheri et al., 2004). Symptom improvement is demonstrated by a lower score on one or more items of the CAHQ. The CAHQ was published in Buccheri et al. (2004).

The UVS measures intensity of auditory hallucinations over 24 hours and over one week on a scale of 0 (no voices heard) to 10 (the most unpleasant the voices could be), presence of pleasant voices, presence of commands to harm self and others and intent to act on those harm commands (Gerlock et al, 2010). Improvement is demonstrated by a lower score on those items measuring intensity of auditory hallucinations (items 1 and 2) and absence for those measuring harms command or intent to act on harm commands (items 4-7). The UVS was published in Gerlock et al. (2010).

Table 1 provides a summary of information about these three instruments. This summary includes a brief description of each instrument including number, type and content of items, how to interpret scores, whether it is used to assess symptoms, measure outcomes or both and references.

\section{Nursing Interventions}

The second part of the model is comprised of both process and content for nursing interventions used in teaching self-management of AH in a 10-session course (or individually if a group is not feasible). This part of the model includes: 1) creating an environment that promotes learning and 2) strategies taught to manage unpleasant $\mathrm{AH}$ (both displayed in column 2 of Figure 1). We consider each of these equally important. 
Facilitators use the Treatment Manual: Guidelines for Teaching the 10-Session Behavioral Management of Auditory Hallucinations Course (Buccheri, Trygstad, \& Buffum, 2012) as an operational guide providing information about process and content for teaching the course. The Treatment Manual provides information about ideal class size, how to set the climate for the course, how to handle issues that may arise and how to maintain safety. The Treatment Manual also gives specific directions for how to teach each class and class content. Content for each class includes the "strategy of the week", the rationale for why the strategy is effective and directions for practicing the strategy during class and at home.

Clinicians have used the Treatment Manual to work with individual voice hearers when having them attend the course is not possible or when conducting a course is not possible in a particular setting. The Treatment Manual also includes Individual Practice Records (IPRs), the AHIG, CAHQ, UVS, course participant and course facilitator feedback forms and a certificate of achievement that is given to course participants upon completion of the course.

Process: creating an environment that promotes learning. Learning among course participants can be facilitated by creating an environment that is conducive to learning. Course facilitators can create an environment that promotes learning by building trust, maintaining safety, providing structure and using consistent teaching methods.

Building trust. Trust by individual course participants and trust within the group are both important to facilitate learning. Trust can be enhanced with individual participants when course facilitators conduct the AHIG to assess the voice hearer's past and current 
experiences with hearing voices. During the interview process, the course facilitator can demonstrate empathy, show interest, and use communication skills that show respect and support. These behaviors facilitate relationship building between the psychiatric-mental health nurse and the voice-hearer. The outcomes from the AHIG interview process are often increased trust and comfort for both course participant and course facilitator.

Course facilitators can encourage the development of trust within the group by maintaining a safe environment, showing respect for each course participant and the group as a whole and being consistent in saying what they will do and following through. The repetitive structure of the group demonstrates consistency and clear expectations, thereby also helping to build trust.

Maintaining safety. The teaching environment including all interactions must be safe. Prompt nursing action is required if any threat to safety occurs. The topic of voices is sensitive and voice hearers may be discussing their $\mathrm{AH}$ for the first time in a group setting. The course facilitators provide an atmosphere where it is acceptable for group members to admit that they hear $\mathrm{AH}$ and talk about their experience of hearing $\mathrm{AH}$. These discussions include describing situations that make AH better or worse.

Forty-seven percent of the voice hearers who participated in one study reported they heard voices to harm self or others (Buccheri et al., 2007). Based on this finding, course participants are asked to complete questions on the UVS that ask if they hear harm voices and whether or not they intend to act on them. If they answer yes to questions about intent to harm self or others, then course facilitators follow a supportive safety protocol (Safety Protocol: Protocol Response to Intent to Harm Self or Others) to guide their 
decision about a need for intervention to keep the course participant safe (Gerlock et al., 2009).

Providing structure. We teach symptom management strategies in a highly structured and supportive education group that optimizes learning opportunities. The agenda for each class is visually displayed on a whiteboard, blackboard or flipchart. This structure decreases anxiety as it helps course participants know what to expect during each class.

At the beginning of the course, course facilitators establish a set of group rules and course participants are welcome to make additions. Group rules are posted in each class and are displayed in Table 2. There is no mandatory requirement that each course participant make a verbal contribution in class. We use minimal silence and supportive redirection to course participants wandering off topic, conveying delusional content, or monopolization. Both professional and peer validation are useful for recognizing, acknowledging, and validating similarities and differences in the individual experience of AH. Course participants are encouraged to stay for the duration of the class but it is acceptable for participants to leave if they feel too anxious to remain; they are welcome to return when ready.

Using supportive teaching methods. We use supportive teaching methods that are respectful, validating, inclusive, and interactive. People with AH are the experts on their own symptom. That is, they know when they experience $\mathrm{AH}$, whether the $\mathrm{AH}$ are pleasant, unpleasant, distressing or distracting and when an intervention is or is not helpful. Many course participants have pleasant voices they think they may wish to retain; we assure them that we respect their choice. We validate the experience of 
distress with unpleasant $\mathrm{AH}$ and also the person's right to choose what/when/where they wish to engage in symptom self-management

Specific concrete teaching (step by step) helps course participants use strategies they have already found useful and to learn new strategies to manage their AH. Cognitive limitations and symptoms of schizophrenia and other mental disorders may limit some participants' ability to internalize from observations and generalize from experience. We employ strategies to overcome problems with cognition (memory, attention span, concentration and thought disorders). For example, to help with memory deficits, we repeat guidelines in simple statements - reviewing them several times. Short, simple directions compensate for a short attention span. Redirection and refocusing help course participants experiencing delusions or disorganized thought processes concentrate on the content being presented.

Education about symptom self-management is a shared learning experience among course participants and course facilitators in the group. Course facilitators express their interest and appreciation for the opportunity to learn from voice hearers. Course participants often share their experience more openly if they know it will help others with unpleasant AH. Course facilitators demonstrate each strategy in the group and have course participants practice the strategy together in the group. IPRs have directions for how to practice the "strategy of the week" twice a day (AM and PM) at home. Practicing the behavioral strategies in each voice hearer's living environment and completing an IPR increases the likelihood that a course participant will incorporate the strategies into their lives (Liberman, 1988). Green and Kinsbourne's (1989) taught subjects to hum in the lab when they heard voices. In the lab, humming was $100 \%$ effective in managing 
the voices but not one subject hummed outside the lab when they heard voices.

Sometimes we are able to provide small rewards/incentives to course participants for completing and returning the IPRs to encourage practice until they experience the benefits of practicing the strategies.

Content: strategies taught to manage unpleasant AH. The strategies taught in the course are listed in Figure 1. Evidence supporting each of the strategies is reviewed in prior work (Buccheri et al, 1996). We highlight and emphasize the importance of the first strategy of "self-monitoring through symptom self-awareness" in the course because of its importance in setting the foundation for the remainder of the course. This strategy is based on the work of Brier and Strauss (1983) who found that all of the patients in their study who had the ability to exert control over their symptoms also had the ability to monitor their own behavior.

The remaining strategies were selected from those in the research literature with the strongest evidence. At each class, the course facilitators describe the new "strategy of the week" and why it may be effective. For example, when the strategy of the week is "talking with someone" participants are told: a) use of voice muscles, eyes and ears are all associated with a reduction in $\mathrm{AH}$ and $\mathrm{b}$ ) talking with someone distracts from focusing on $\mathrm{AH}$ and changes the focus of concentration.

Course participants are encouraged to make their own decision to stop practicing any strategy that makes them uncomfortable and to use a strategy they are comfortable with in that situation in order to feel safe (e.g. headphones covering the ears are uncomfortable for many paranoid voice hearers and so listening to music with a radio is an encouraged substitution). 


\section{Voice Hearer's Expected Positive Outcomes}

The third part of the model is comprised of two expected positive outcomes: 1) voice hearer selection and use of strategies that work best for them to self-manage $\mathrm{AH}$ and 2) AH symptom improvement. To evaluate the patient's selection and use of selfmanagement strategies, each participant identifies the strategies that work best for them at the end of the course on the Course Participant Feedback Form. AH symptom improvement is measured over time (i.e., during each class, pre and post course) with two specific symptom outcome measures: the Characteristics of Auditory Hallucinations (CAHQ) and the Unpleasant Voices Scale (UVS/0-10). Voice hearers usually show improvement on CAHQ and UVS scores as they become proficient with their selected self-management strategies. These scores are valuable for voice hearer education and discussion about the success of symptom self-management and measurement of progress over time. Both of these instruments are described in the Assessment of Voice Hearer's Experience and summarized in Table 1.

Two evaluation instruments (Course Participant Feedback Form and Course Facilitator Feedback Form) provide feedback about the course. The Course Participant Feedback Form is a subjective appraisal of course helpfulness, willingness to take the course again, and willingness to refer a friend. The Course Facilitator Feedback Form appraises helpfulness of each element in the course, benefit to course participants, and intent to teach the course again. Both course participants and course facilitators are encouraged to write-in their opinions, experiences, obstacles and successes, course strengths, and suggestions for course improvement on these evaluation forms. 


\section{Application of the Model}

\section{Clinical Practice Settings}

The 10-session Behavioral Management of Auditory Hallucinations Course has been taught widely in the USA in both Veterans Affairs (VA) and nonVA settings and in 6 other countries (i.e., Australia, Canada, Thailand, Taiwan, India, Japan). The authors (LT, RB \& MB) first taught the course themselves in varied types of inpatient and outpatient settings. Subsequently, they realized more voice hearers could benefit if they taught other mental health clinicians to teach the course. So they taught Advanced Practice Nurses (APNs) how to teach the course; results indicated no differences in patient outcomes.

The authors have conducted many dissemination research projects where they have taught a variety of mental health clinicians (e.g., psychiatric-mental health nurses, psychologists, social workers, psychiatrists) how to teach the course either in person or more recently by regularly scheduled teleconference sessions and e-mail correspondence. As a result of the authors' trainings, clinicians have implemented the course in their clinical practice settings that have included short and long-term inpatient units and many different types of outpatient rehabilitation programs. These clinicians have also taught the content to individual voice hearers when they are unwilling or unable to attend a group or when a course for multiple patients is not feasible. Once research was completed that tested the benefit of course attendance on patient outcomes, course facilitators were encouraged to modify the course format to meet the needs of their setting (e.g., type of program, patient population and acuity, staff resources, and facility support). 
This model provides consistency for psychiatric-mental health nurses in practice settings by including both tools and teaching approaches for: 1) assessing the voice hearer's experience including onset history, past/current AH experience, characteristics

of $\mathrm{AH}$, presence of pleasant $\mathrm{AH}$, intensity of $\mathrm{AH}$ over 24 hours and one week, presence of harm commands and intent to act on those commands; 2) creating and maintaining a safe environment in which to teach evidence-based symptom self-management strategies either in a 10 -session course or individually and 3) measuring voice hearer outcomes with the CAHQ and the UVS after attendance at the 10-session course or individualized teaching.

\section{Academic settings}

This practice model offers a framework for teaching both undergraduate and graduate nursing students how to work therapeutically with people who hear unpleasant AH. Faculty can promote student awareness of the voice hearer's experience of AH by having them use the AHIG to establish rapport with voice hearers, teaching them the characteristics of $\mathrm{AH}$ and which situations may lessen the intensity of $\mathrm{AH}$ (e.g., talking with someone, helping someone) and increase the intensity of $\mathrm{AH}$ (e.g., crowded bus, stress, street drugs). Faculty can teach students how to administer the CAHQ and the UVS to assess the characteristics and intensity of each voice hearer's unpleasant AH, presence of harm commands, intent to act on harm commands and how to implement the safety protocol. Faculty can also supervise students as they teach self-management strategies to a group of voice hearers in the 10-session course or teach an individual voice hearer self-management strategies, measure outcomes of these interventions with the CAHQ and UVS and communicate outcomes to staff. 


\section{Case Study}

The following case study demonstrates application of this practice model and how psychiatric-mental health nurses can use it to improve care of patients with unpleasant

\section{AH.}

\section{Encountering the Problem}

On an inpatient psychiatric unit, Susan, a psychiatric-mental health staff nurse is concerned about a male patient who is tormented by hearing unpleasant $\mathrm{AH}$ and has a history of self-harm. Susan says in report: "All I can do is ask him if he hears voices, ask if they tell him to harm himself, try to keep him safe by monitoring his behavior and offer him his ordered PRN of antipsychotic or anti-anxiety medication. There must be more that I can do!"

\section{Using Evidence to Address the Problem}

Susan brings up her concerns about not being able to do enough for her patient with unpleasant AH in the next unit meeting. Susan's nurse manager recommends that she take the issue to the unit's Journal Club. Susan reviews the literature before attending the Journal Club and finds this article about a new practice model for teaching selfmanagement of unpleasant $\mathrm{AH}$ and brings copies of it to the Journal Club. Members of the Journal Club discuss Susan's concerns and agree to do two things before their next meeting: 1) read this article so they can critique it during their next meeting and 2) try using one of the tools in the article-the Auditory Hallucinations Interview Guide (AHIG) and report what they learn at the next meeting. At the next Journal Club meeting, Susan reports what it was like to interview a patient using the AHIG. Susan tells the Journal Club members that the interview process served to promote a bond 
between herself and the patient. The patient told Susan that he felt comfortable for the first time talking about hearing voices. Other members also shared their experiences using the AHIG and the decision was made to explore implementation of this evidencebased practice model on the unit for patients who hear AH. To provide background for the practice model and support for the evidence, another Journal Club member offered to present the same authors' articles from prior research. After this was done, the members of the Journal Club decided that there was enough evidence to recommend implementing this practice model on their unit. Members of the Journal Club presented their evidence at the next unit meeting and recommended implementing this practice model including teaching the 10-Session Behavioral Management of Auditory Hallucinations Course on the unit. The decision was made by administrators and staff to implement the model. Implementing the model:

\section{Assessment of the patient experience:}

Susan is excited to assess a patient using this practice model and uses the Auditory Hallucinations Interview Guide (AHIG) to interview a female patient with AH. Susan learns that the patient's voices are loudest and most distressing before bedtime and that music helps distract her from them. The patient says she hears only one voice, her neighbor's, who molested her as a child; the voice now threatens to hurt the patient's family. The patient often feels that she should do what the voices tell her to do--harm herself. The patient had a history of one suicide attempt 2 years prior to this admission. Susan then reviewed the Unpleasant Voices Scale (UVS/0-10) with the patient, and learned that the patient's symptom severity was 8 of 10 over the past 24 hours, that she heard self-harm commands, and that she was fearful of responding aloud to the 
commands and embarrassing herself in public places. The patient stated that she did not intend to act on the harm commands. Teaching the patient to complete the Characteristics of Auditory Hallucinations Questionnaire (CAHQ), Susan learned about the frequency, loudness, self-control, clarity, tone, distractibility, and distress of her AH. Using information she learned from doing the AHIG interview and having the patient complete the UVS and CAHQ, Susan planned evidence-based and customized interventions and included them in an individualized care plan for this patient. Nursing Interventions: Because the patient was fearful and had a history of self-harm, Susan was prepared to implement the Safety Protocol: Protocol Response to Intent to Harm Self or Others if the patient's intent to act on harm commands changed. The patient was encouraged to attend a new course that was about to start on the unit: "10-Session Behavioral Management of Auditory Hallucinations Course". The course was cofacilitated by two psychiatric-mental health nurses who used a structured format to create a safe environment where they could help course participants learn, discuss and practice strategies to self-manage their unpleasant voices.

Patient Outcomes: The patient attended the 10-session course and expressed great relief to the course facilitators that she was not the only one who heard voices. She shared how music had helped her manage her unpleasant $\mathrm{AH}$. The patient liked being with the other patients when outside of the group; this enabled her to help others, a new strategy for her. She also discovered that chewing gum helped her; this was a strategy another patient shared in class. The patient's UVS/0-10 scores improved after attendance at the class and she had no self-harm incidents during her hospital stay. The patient was discharged after 10 days with multiple resources: a list of her 3 preferred strategies, a plan for how to 
communicate about voices to her case manager and her family, and a plan for what to do when she heard voices during her volunteer job. Susan reported back to the Journal Club that since implementing this practice model, she felt that they were doing a lot more to help their patients with unpleasant voices.

\section{Implications and Conclusions}

Approximately two decades ago, we created a 10-session course to teach behavioral management of AH that was developed from theories and research findings. We taught the course and evaluated patient outcomes over time (i.e., characteristics and intensity of $\mathrm{AH}$, presence of commands to harm and intent to act on those commands, anxiety and depression). We disseminated the course widely in the USA and in 6 other countries and found that others can teach the course with the same patient benefits. Facilitators' ease of course implementation has reinforced replication until the course has become the standard of care in many mental health programs. Based on feedback from course facilitator and course participant evaluations, the 10 -session course has steadily improved.

In this paper, we present a practice model of our work to make clear each part of the model: assessment, interventions and outcomes. This model helps to keep our work focused on testing each part of the model. Because the model serves as a written and visual guide for the 10 -session course, the model has been instructive for introducing the evidence-based course to psychiatric-mental health nurses interested in teaching the course.

The resemblance of the model to the nursing process contributes to the ease of teaching it to students and practicing nurses. Indeed, assessment, intervention, and 
evaluation of outcomes is the way that nurses have learned to approach clinical problems. Further, the inter-relatedness between the model's elements can also be explained using Holzemer's (1994) adaptation of Donabedian's structure-process-outcomes model for nursing in which course participants, facilitators, and physical environment comprise the structure; all course components and interactions comprise the process; and patients and facilitators contribute to the outcomes. We continue to evaluate the interactions between structure-process-outcome, such as the impact of facilitator discipline, number of sessions needed for teaching the strategies, and setting-specific variations from reported format modifications.

Clinical utility, feasibility and demonstrated patient improvement have contributed to the 10 -session course's international popularity. Certainly, evidence-based practice that teaches the benefit of self-management strategies for reducing distress from unpleasant AH deserves wide dissemination. We are hopeful that this model will provide guidance for wider implementation. We expect that this model, which is supported by our past research findings, will continue to evolve with findings from future course implementation experiences and help us to continue to revise our work, test revisions and incorporate improvements to share with others.

Based on our findings, we are moving to the next phase of our work. We have noted the impact of anxiety on our course participants and created a 12-session course that begins by focusing on anxiety management then threads anxiety management throughout the course. If our research demonstrates patient benefit, we will alter this model to reflect new findings. 
We always invite readers to contact us to teach the evidence-based 10-session course or to join us in our research on evaluating the effect of the 12-session course on patient outcomes. If you are interested in teaching either the 10-session or 12-session course, please contact Dr. Robin Buccheri at buccherir@usfca.edu. 


\section{References}

Brier, G., \& Strauss, J. (1983). Self-control in psychotic disorders. Archives of General Psychiatry, 40, 1141-1145.

Buccheri, R., Trygstad, L., \& Buffum, M. (2012). Treatment Manual: Guidelines for Teaching the 10-Session Behavioral Management of Auditory Hallucinations Course.

Buccheri, R., Trygstad, L., \& Dowling, G. (2007). Behavioral management of command hallucinations to harm in schizophrenia. Journal of Psychosocial Nursing and Mental Health Services, 45(9), 46-54.

Buccheri, R., Trygstad, L., Dowling, G., Hopkins, R., White, K., Griffin, J., et al. (2004). Long term effects of teaching behavioral strategies for managing persistent auditory hallucinations in schizophrenia. Journal of Psychosocial Nursing, 42(1), 18-27.

Buccheri, R. K., Trygstad, L., Kanas, N., \& Dowling, G. (1997). Symptom management of auditory hallucinations in schizophrenia: Results of 1-year follow up. Journal of Psychosocial Nursing and Mental Health Services, 35(12), 20-28.

Buccheri, R. K., Trygstad, L., Kanas, N., Waldron, B., Dowling G. (1996). Auditory hallucinations in schizophrenia: Group experience in examining symptom management and behavioral strategies. Journal of Psychosocial Nursing and Mental Health Services, 34, 12-25.

Buffum, M., Buccheri, R., Trygstad, L., Gerlock, A., Birmingham, P., Dowling, G. A., \& Kuhlman, G. (2009). Implementing evidence-based practice: Educating nurses to conduct the behavioral management of auditory hallucinations course. Journal of Psychosocial Nursing and Mental Health Services, (47(9), 32-40. 
Davidson, L., Chinman, M. J., Kloos, B., Weingarten, R., Stayner. D. A., \& Tebes. J. K. (1999). Peer support among individuals with severe mental illness: A review of the evidence. Clinical Psychology: Science and Practice, 6(2), 165-187.

Deegan, P. (1988). Recovery: The lived experience of rehabilitation. Psychosocial Rehabilitation Journal, 11(4), 47-54.

Dodd, M., Janson, S., Facione, N. Faucett, J. Froelicher, E. S., Humphreys, J., Lee, K. Miaskowski, C, Puntillo, K., Rankin, S. \& Taylor, D. (2001). Advancing the science of symptom management. Journal of Advanced Nursing, 33(5), 668-676.

Gerlock, A., Buccheri, R., Buffum, M., Trygstad, L., \& Dowling, G. (2009). Responding to command hallucinations to harm: The Unpleasant Voices Scale and Harm Command Safety Protocol. Journal of Psychosocial Nursing and Mental Health Services, 48(5), 26-33.

Green , M. F., \& Kinsbourne, M. (1989). Auditory hallucinations in schizophrenia: Dues humming help? Biological Psychiatry, 25, 630-633.

Holzemer, W. L. (1994). The impact of nursing care in Latin America and the Carribbean: A focus on outcomes. Journal of Advanced Nursing, 20, 5-12

Humphreys, J., Lee, K. A., Carrieri-Kohlman, V., Puntillo, K. Faucett, J. Janson, S. , Aouizerat, B., Donesky-Cuenco, D. \& the UCSF School of Nursing Symptom Management Faculty Group. (2008). Theory of Symptom Management. In M. J. Smith, \& P. R. Liehr (Eds.), Middle Range Theory of Nursing (pp. 145-158). New York: Springer Publishing.

Kanfer, F. H. (1971). The maintenance of behavior by self-generated stimuli and reinforcement. In A. Jacobs, \& L. B. Sachs (Eds.), The psychology of private 
events: Perspectives on covert response systems (pp. 39-59). New York: Academic Press.

Knowles, M. (1973). The adult learner: A neglected species $\left(2^{\text {nd }}\right.$ ed.). Houston, TX: Gulf Publishing.

Knowles, M. (1998). The adult learner: The definitive classic in adult education and human resource development. Houston, TX: Gulf Publishing.

Liberman, R. P. (1988). International perspectives on skills training for the mentally disabled. International Review of Psychiatry, 10, 5-8.

Romme, M. A. J., \& Escher, A. D. M. A C. (1989). Hearing voices. Schizophrenia Bulletin, 15(2) 209-216.

Romme, M. (2009). The disease concept of hearing voices and its harmful aspect. In M. Romme, S. Escher, J. Dillon, D. Cortens, \& M. Morris (Eds.), Living with Voices: 50 stories of recovery (p. 24). Herefordshire, UK: PCCS Books.

Shinn, A. K., Pfaff, D., Young, S., Lewandowski, K. E., Cohen, B. M., Öngür, D. (2012). Auditory hallucinations in a cross-diagnostic sample of psychotic disorder patients: A descriptive, cross-sectional study. Comprehensive Psychiatry, 53(6), 718-726.

Trygstad L., Buccheri, R., Dowling, G., Zind, R., White, K., Griffin, J., et al. (2002). Behavioral management of persistent auditory hallucinations in schizophrenia: Outcomes from a 10-week course. Journal of the American Psychiatric Nurses Association, 8(3), 84-91.

UCSF Symptom Management Faculty Group. (1995). A model for symptom management. The Journal of Nursing Scholarship, 26, 272-276. 


\section{Figure 1. Self-Management of Unpleasant Auditory Hallucinations (AH): A Tested Practice}

Model

Assessment of

Voice Hearer's Experience

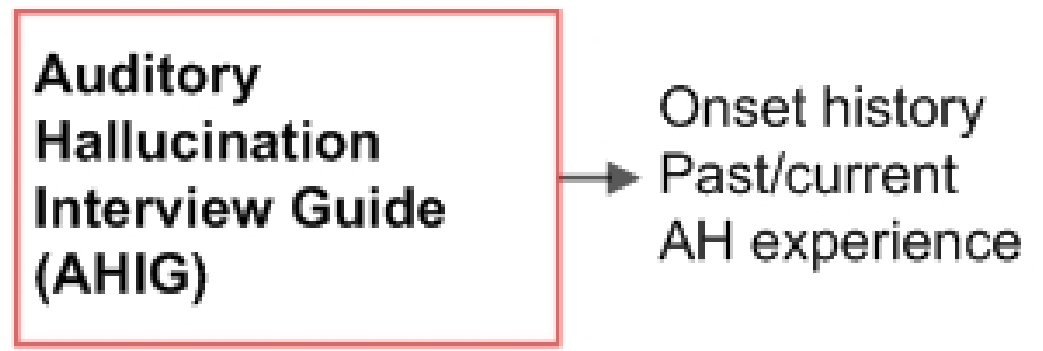

\begin{tabular}{|l|l|l}
\hline $\begin{array}{l}\text { Characteristics } \\
\text { of AH }\end{array}$ & $\begin{array}{l}\text { Frequency } \\
\text { Loudness } \\
\text { Questionnaire } \\
\text { (CAHQ) }\end{array}$ & Self-control \\
\cline { 2 - 2 } & Clarity \\
& Tone \\
& Distractibility \\
& Distress
\end{tabular}

\begin{tabular}{|l|l|}
\hline & $\begin{array}{l}\text { Intensity } \\
\text { Unpleasant Voices } \\
\text { Scale (UVS) 0-10 }\end{array}$ \\
\cline { 2 - 2 } & $\begin{array}{l}\text { Presence of } \\
\text { harm commands } \\
\text { Intent to act on } \\
\text { harm commands }\end{array}$ \\
&
\end{tabular}

Nursing Interventions

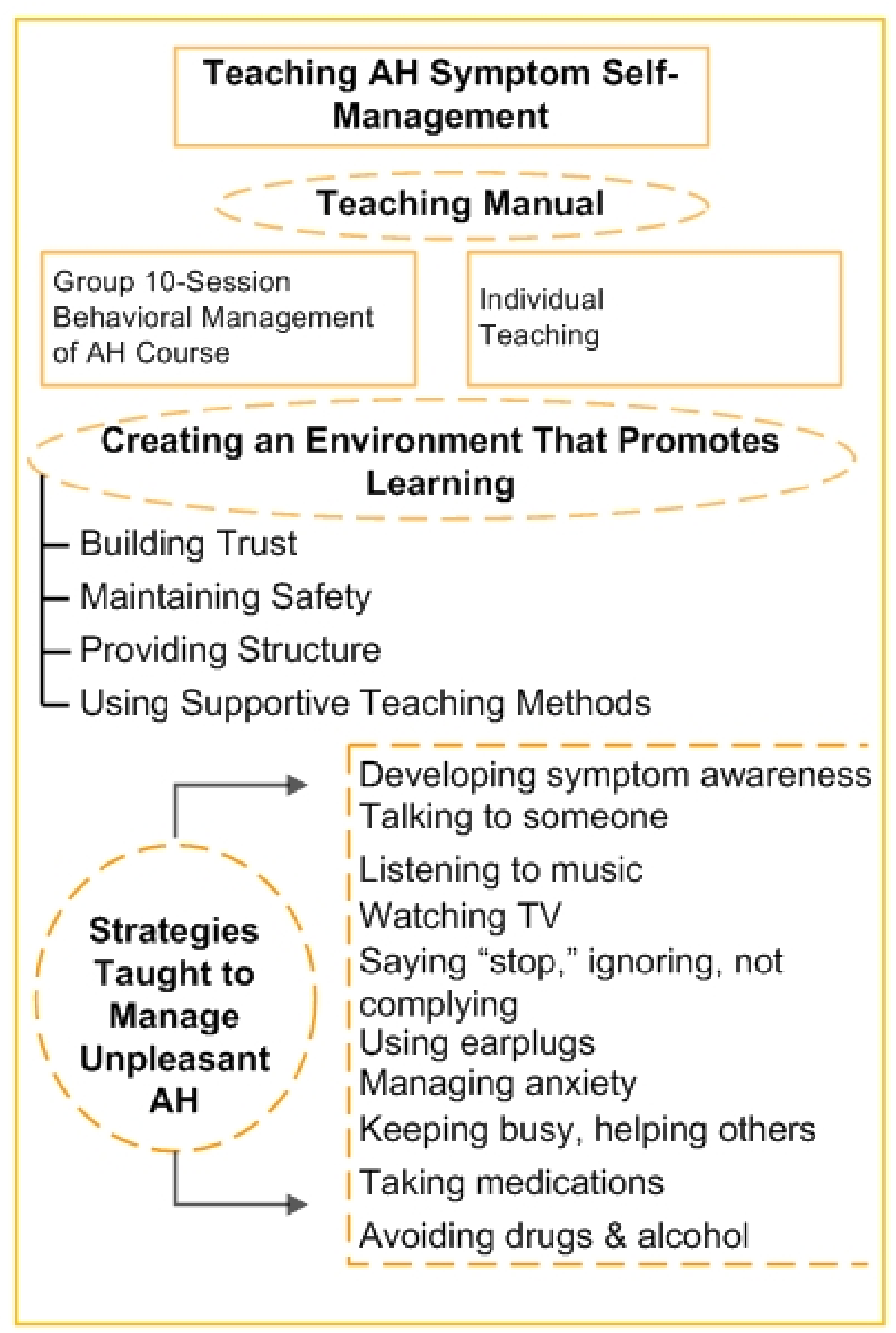

Voice Hearer's Expected Positive Outcomes

Individuals Select and Use Strategies That Work Best for Them

AH Symptom Improvement CAHQ/UVS 0-10

\section{Self-Management of $\mathrm{AH}$}

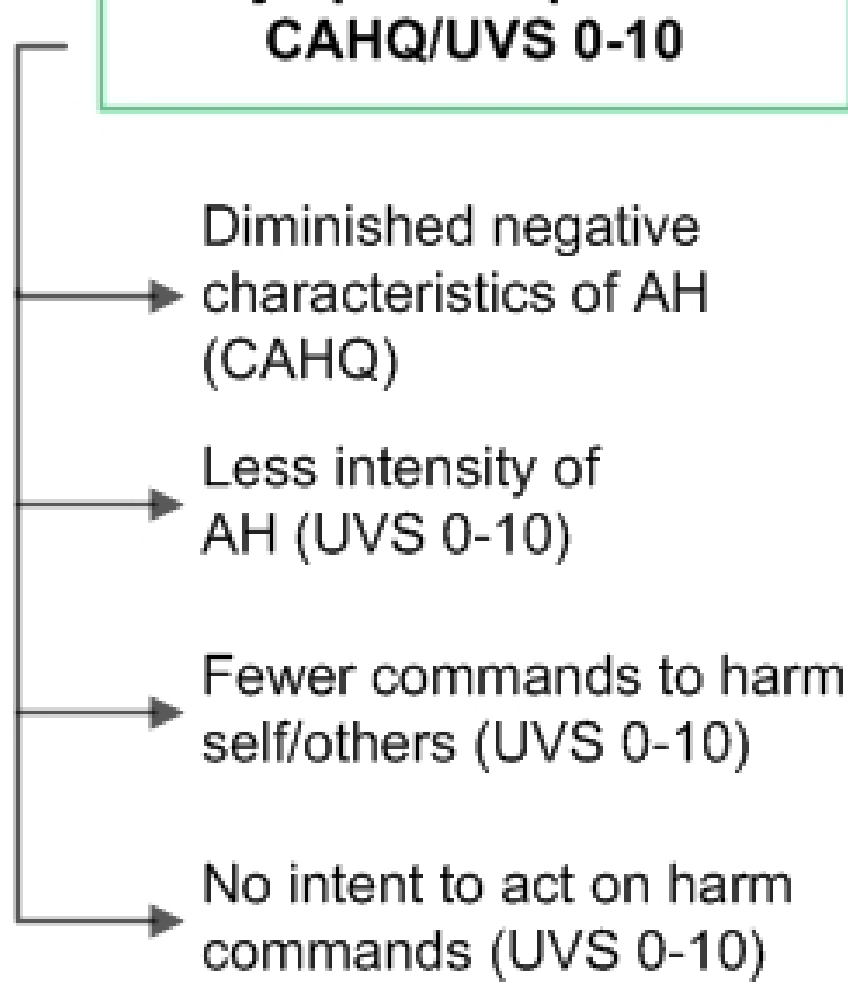


Table 1 Instruments for Assessment of Voice Hearer's Experience and Outcome Measurement

\begin{tabular}{|c|c|c|c|c|}
\hline Instrument & Description & $\begin{array}{l}\text { Used in } \\
\text { Assessment } \\
\text { of Voice } \\
\text { Hearer's } \\
\text { Experience }\end{array}$ & $\begin{array}{l}\text { Used to } \\
\text { Measure } \\
\text { Voice } \\
\text { Hearer's } \\
\text { Outcomes }\end{array}$ & References \\
\hline $\begin{array}{l}\text { Auditory } \\
\text { Hallucinations } \\
\text { Interview Guide } \\
\text { (AHIG) }\end{array}$ & $\begin{array}{l}\text { yes/no and open-ended } \\
\text { questions that ask about: } \\
\text { - circumstances } \\
\text { surrounding onset } \\
\text { - description of current } \\
\text { and past experience of } \\
\text { hearing voices } \\
\text { - hearers' own words } \\
\text { for what helps them } \\
\text { manage their voices }\end{array}$ & $\mathrm{x}$ & & $\begin{array}{l}\text { Buccheri, } \\
\text { Trygstad, Kanas, } \\
\text { Waldron, \& } \\
\text { Dowling, 1996; } \\
\text { Buccheri, } \\
\text { Trygstad \& } \\
\text { Dowling, 2007; } \\
\text { Buccheri, } \\
\text { Trygstad, Buffum, } \\
\text { Lyttle, \& } \\
\text { Dowling, } 2010\end{array}$ \\
\hline $\begin{array}{l}\text { Characteristics of } \\
\text { Auditory } \\
\text { Hallucinations } \\
\text { Questionnaire } \\
\text { (CAHQ) }\end{array}$ & $\begin{array}{l}\text { 7-item Likert-type scale } \\
\text { that measures: } \\
\text { - characteristics of AH } \\
\text { (frequency, loudness, } \\
\text { self-control, clarity, } \\
\text { tone, distractibility } \\
\text { and distress) } \\
\text { Symptom } \\
\text { improvement=lower } \\
\text { scores on items }\end{array}$ & $\mathrm{x}$ & $\mathrm{x}$ & $\begin{array}{l}\text { Buccheri et al., } \\
\text { 2004, 2007, 2010; } \\
\text { Buffum et al., } \\
\text { 2009; Trygstad et } \\
\text { al., 2002 }\end{array}$ \\
\hline $\begin{array}{l}\text { Unpleasant } \\
\text { Voices Scale } \\
(U V S / 0-10)\end{array}$ & $\begin{array}{l}\text { 7-item (2-item Likert- } \\
\text { type scale and } 5 \text { yes/no } \\
\text { items) that measure: } \\
\text { - unpleasant voices over } \\
\text { the "past } 24 \text { hours" } \\
\text { and "past week" } \\
\text { - presence of pleasant } \\
\text { voices, } \\
\text { - presence of harm } \\
\text { commands } \\
\text { - intent to act on harm } \\
\text { commands } \\
\text { Symptom } \\
\text { improvement=lower } \\
\text { scores on items } 1 \& 2 \\
\text { and more "no" } \\
\text { responses on items 4-7 }\end{array}$ & $\mathrm{x}$ & $\mathrm{x}$ & $\begin{array}{l}\text { Buccheri et al., } \\
\text { 2007, 2010; } \\
\text { Buffum et al, } \\
\text { 2009; Gerlock, } \\
\text { Buccheri, Buffum, } \\
\text { Trygstad, \& } \\
\text { Dowling, } 2010\end{array}$ \\
\hline
\end{tabular}


Table 2 Group Rules for 10-Session Behavioral Management of

Auditory Hallucinations Course

1. Classes will start and end on time.

2. One person talks at a time.

3. Everyone gets a chance to talk. No one is required to talk.

4. Be respectful and courteous to others. Can disagree but not get angry at others. Must present issue politely. (Critique the idea not the person.)

5. Nobody talks about someone else's experience outside the group. (What is said in here stays in here.)

6. Try to stay for entire class but may leave if needed. It is OK to return.

7. Cell phone calls are not acceptable in class. 